\title{
A comparison of observation-level random effect and Beta- Binomial models for modelling overdispersion in Binomial data in ecology \& evolution
}

Xavier A Harrison

Overdispersion is a common feature of models of biological data, but researchers often fail to model the excess variation driving the overdispersion, resulting in biased parameter estimates and standard errors. Quantifying and modeling overdispersion when it is present is therefore critical for robust biological inference. One means to account for overdispersion is to add an observation-level random effect (OLRE) to a model, where each data point receives a unique level of a random effect that can absorb the extra-parametric variation in the data. Although some studies have investigated the utility of OLRE to model overdispersion in Poisson count data, studies doing so for Binomial proportion data are scarce. Here I use a simulation approach to investigate the ability of both OLRE models and Beta-Binomial models to recover unbiased parameter estimates in mixed effects models of Binomial data under various degrees of overdispersion. In addition, as ecologists often fit random intercept terms to models when the random effect sample size is low $(<5$ levels), I investigate the performance of both model types under a range of random effect sample sizes when overdispersion is present. Simulation results revealed that the efficacy of OLRE depends on the process that generated the overdispersion; OLRE failed to cope with overdispersion generated from a Beta-Binomial mixture model, leading to biased slope and intercept estimates, but performed well for overdispersion generated by adding random noise to the linear predictor. Comparison of parameter estimates from an OLRE model with those from its corresponding Beta-Binomial model readily identified when OLRE were performing poorly due to disagreement between effect sizes, and this strategy should be employed whenever OLRE are used for Binomial data to assess their reliability. Beta-Binomial models performed well across all contexts, but showed a tendency to underestimate effect sizes when modelling non-Beta-Binomial data. Finally, both OLRE and Beta-Binomial models performed poorly when models contained $<5$ levels of the random intercept term, especially for estimating variance components, and this effect appeared independent of total sample size. These results suggest that OLRE are a useful tool for modelling overdispersion in Binomial data, but that they do not perform well in all circumstances and researchers should take care to verify the robustness of parameter 
estimates of OLRE models. 
1 Xavier A. Harrison ${ }^{1}$

$2{ }^{1}$ Institute of Zoology, Zoological Society of London, Regent's Park, NW1 4RY, UK 3

4 


\section{INTRODUCTION}

7 Binomial data are frequently encountered in the fields of ecology and evolution. Researchers

8 often wish to know what factors determine the proportion of offspring sired by a focal

9 individual (Tyler et al 2013), the proportion of eggs of a clutch that successfully hatch (Harrison et al 2013a), or the prevalence of disease in a population (Bielby et al 2014). To determine which factors drive variation in the proportion data of interest, researchers often fit Binomial models to their data and model the Binomial mean as a function of covariates. However, in many cases these Binomial models exhibit overdispersion, where the variance of the data is greater than that predicted by the model (e.g. Zuur et al 2009; Bolker et al 2009). Failing to deal with overdispersion can lead to biased parameter estimates and standard errors in these models (Hilbe 2011; Harrison 2014), potentially leading to false conclusions regarding which covariates are truly influential on the outcome variable. It is therefore crucial that we find robust means to deal with overdispersion in order to correctly identify the biological processes underlying our observed Binomial data.

Several methods to deal with overdispersion are currently available. As overdispersion can downwardly bias standard errors in models, one method involves 'correcting' the standard errors by multiplying them by the square root of the dispersion coefficient (Zuur et al 2009). This multiplicative correction for overdispersion occurs when one specifies the 'quasi' family in Generalized Linear Models (GLMs) in the statistical software R (R Core Team 2014). However, a weakness of the 'quasi' approach is that it does not model the overdispersion in the data, but merely adjusts the resulting parameter estimates with a single correction factor. The assumption that all standard errors are biased to the same degree is an obvious problem, which may not be appropriate (e.g. Harrison 2014 Table 1). The alternatives to the 'quasi' approach for proportion data are to explicitly model the source of extra-Binomial variation in the data (e.g. Williams 1982; Hughes and Madden 1993; Lee and Nelder 1996; Clark 2003; Richards 2008), for example by using compound probability structures (e.g. Beta-Binomial models), or to use observation-level random effects (OLRE). With OLRE models, each observation in the model receives a unique level of a random effect that absorbs the extra-Binomial variation in the data, 
34 hopefully yielding a model with unbiased parameter estimates and without overdispersion.

35 However, although several studies have sought to investigate the utility of OLRE to model

36 overdispersion in Poisson count data (Elston et al 2001; Harrison 2014), similar investigations

37 for Binomial proportion data are relatively rare. Harrison (2014) found that for Poisson data,

38 OLRE yielded accurate parameter estimates and $r^{2}$ values in most situations of overdispersion, 39 but that OLRE could not adequately cope with overdispersion caused by zero-inflation. Here I

40 will address the shortfall in our understanding of the capacity of OLRE to model overdispersion

41 in Binomial data, with a specific focus on mixed effects models. In order for OLRE to be an

42 appropriate tool, they should be robust to the process generating overdispersion in the data, 43 and thus I test OLRE on overdispersed Binomial data generated by a variety of mechanisms. In 44 addition, I explore the utility of Beta-Binomial hierarchical models as an alternative to OLRE 45 models, and compare the accuracy of parameter estimates derived from both approaches.

\section{A Typical Binomial Example}

48

49

53

54

55

56

57

61

62

For these examples, I will assume the outcome variable that we are measuring is the number of eggs $h$ that have hatched out of a total clutch $c$ for a hypothetical lizard species. I assume that the proportion of hatched eggs is described by a Binomial distribution:

$$
h_{i} \sim \operatorname{Binomial}\left(c_{i}, p_{i}\right)
$$

where $h_{i}$ is the number of eggs hatched by individual i from its total clutch $\mathrm{c}_{\mathrm{i}}$, with mean probability $p_{i .}$ In a typical Binomial model, we can model the mean hatch rate $p_{i}$ as a function of covariates of interest. Let us assume that hatch rate shows a positive relationship with how many prey items an individual lizard has consumed, and also that there is a weak negative relationship between body size and hatch rate. Let us also assume we have measured $N$ individuals from J populations of lizards, and that we wish to control for variation among populations using a random intercept.

$$
\operatorname{logit}\left(p_{i}\right)=\operatorname{alpha}_{j(i)}+\beta_{\text {prey }} \times \text { Prey }_{i}+\beta_{\text {bodysize }} \times \text { Bodysize }_{i}
$$


63

64

65

66

67

68

69

70

71

72

73

74

75

76

77

78

79

80

81

82

83

84

85

86

87

88

89

90

where the hatch rate $p_{i}$ is a function of a linear model. $a l p h a_{j(i)}$ is the intercept for population $j$

to which individual $i$ belongs, where each $a l p h a_{j}$ is drawn from a normal distribution with mean $\mu_{\text {pop }}$ and variance $\sigma_{\text {pop }}^{2}$ (Eqn. 3). $\beta_{\text {prey }}$ and $\beta_{\text {bodysize }}$ are the slope parameters for the effects of number of prey items consumed and body size, respectively. Prey ${ }_{i}$ and Bodysize $e_{i}$ are the prey and body size measurements of individual $i$. We convert the linear predictor (logit. $p_{\mathrm{f}}$ from, Eqn. 1) back to a probability $\left(p_{i}\right)$ using a logit link (Eqn. 4). Suppose now we wanted to model our hatching success data using these covariates. Statistical packages such as Ime4 (Bates et al 2014) readily fit such generalized linear mixed models (GLMMs)

m1<-glmer(cbind(hatch, clutch-hatch) Prey + Bodysize + (1|Population),family=binomial(logit))

$R$ Code

where hatch and clutch are vectors where each row corresponds to the measurements for a single individual $i$ for $h_{i}$ and $c_{i}$ from Eqn 1. Prey and Bodysize are vectors of measurements of Prey $_{i}$ and Bodysize $e_{i}$ corresponding to Eqn. 2. Population is a vector denoting the population ID of each individual. We also specify the Binomial error distribution with a logit link using the 'family' argument in the glmer call. When we fit model $m 1$, we are modeling our data according to equations 1-4 above. The model will estimate $\beta_{\text {prey, }} \beta_{\text {bodysize, }} \mu_{\text {pop }}$, and $\sigma_{\text {pop. }}$. In order to be confident that the resulting parameter estimates are robust, we should check for overdispersion in model $m 1$. Bolker et al (2009) and Harrison (2014) provide R code to calculate the dispersion parameter for such models. Briefly, a point estimate of the dispersion parameter can be calculated as the ratio of the sum of squared Pearson residuals to the residual degrees of freedom for the model, where a value $>1$ indicates overdispersion (Zuur et al 2009, Bolker et al 2009). Harrison (2014) also provides code to estimate the dispersion parameter and $95 \%$ confidence intervals using parametric bootstrapping. If the data exhibit overdispersion, we can 
91 adjust our model to take this into account, either with observation-level random effects, or 92 using a compound error structure such as the Beta-Binomial.

93

94

95 96

97

98

100

101

102

103

104

105

106

107

108

109

110

111

112

113

114

115

116

117

118

119

\section{Modeling Overdispersion Using Observation-Level Random Effects}

Including an observation-level random effect requires that we modify Eqn. 2 above to include an additional term in the linear predictor:

$$
\begin{gathered}
\operatorname{logit}\left(p_{i}\right)=\operatorname{alpha}_{j(i)}+\beta_{1 \text { prey }} \times \text { Prey }_{i}+\beta_{\text {bodysize }} \times \text { Bodysize }_{i}+\varepsilon_{i} \\
\varepsilon_{i} \sim \operatorname{Normal}\left(0, \sigma_{\varepsilon}^{2}\right)
\end{gathered}
$$

where $\varepsilon_{\mathrm{i}}$ is an additional term unique to each observation $i$ that is drawn from a normal distribution with a mean of 0 and variance $\sigma_{\varepsilon}^{2}$. If a dataframe $D$ containing the observations has $N$ rows, we can create an observation level random effect as follows:

obs<-seq(nrow(D))

$R$ Code

where $D$ is the dataframe in which the values of Prey and Bodysize are stored. We can then modify our model $m 1$ to include the OLRE denoted by 'obs':

$$
\begin{gathered}
\text { M2<-glmer(cbind(hatch,clutch-hatch) } \sim \text { Prey + Bodysize + (1|Population } \\
+(1 \mid \text { obs }), \text { family=binomial(logit }))
\end{gathered}
$$

Model $m 2$ will estimate the same parameters as $m 1$, but in addition will also estimate the additional parameter $\sigma_{\varepsilon}^{2}$. The larger the value of $\sigma_{\varepsilon}^{2}$, the greater the degree of overdispersion in the dataset. The magnitude of the variance parameter $\sigma_{\varepsilon}^{2}$ can be informative, for example when compared to hierarchical variance components (e.g. individual nested within brood, nested within site (see Elston et al 2001)). However, in many cases the OLRE will simply 'soak up' the extra-Binomial variation in the data, effectively treating the overdispersion as nuisance variation. The problem with this approach is that often the overdispersion might be biologically 
120 interesting (Zuur et al 2009) and indeed relevant to our hypotheses regarding the processes

121 underlying variation in the observed data. One means by which we can achieve this is by using

122 hierarchical models such as Beta-Binomial models.

123

124 Modeling Overdispersion Using Hierarchical Beta-Binomial Models

125 An alternative to adding an observation-level random effect to models involves model the

126 overdispersion using compound probability distributions such as the Beta-Binomial. The benefit

127 of this approach is that by quantifying the process generating the overdispersion (through the

128 estimate of $\phi$, see below), one may gain a more precise understanding of the ecological

129 mechanisms underlying observed data (Martin et al 2005; Richards 2008). For Beta-Binomial

130 models, the linear predictor remains the same as eqn. 2 - 4 above, on the logit scale, but

131 instead of drawing observed counts directly from a Binomial distribution with mean $p_{i}$, we draw

132 the Binomial probabilities from a beta distribution with parameters $a$ and $b$ :

133

beta. $p_{i} \sim \operatorname{Beta}\left(a_{i}, b_{i}\right)$

134

$$
a_{i}=\frac{p_{i}}{\phi}
$$

$$
b_{i}=\frac{\left(1-p_{i}\right)}{\phi}
$$

$$
h_{i} \sim \text { Binomial }\left(c_{i}, \text { beta. } p_{i}\right)
$$

137

where $a_{i}$ and $b_{i}$ are the shape and scale parameters of the Beta distribution for individual $i$,

calculated using the value of $p_{i}$ (Eqn. 4) and $\phi$, which is the constant overdispersion term in the model. As with $\sigma_{\varepsilon}^{2}$ for the OLRE models above, the larger the value of $\phi$ the greater the degree

141 of overdispersion in the data.

142

\section{Overdispersion and Sample Size}

144 Multiple features biological data can influence the accuracy with which models recover

145 parameter estimates for effect sizes. Overdispersion is likely a ubiquitous feature of the kinds of

146 'messy' data collected by ecologists from field and laboratory studies, and is known to bias

147 parameter estimates in count data (Hilbe 2011; Harrison 2014). In addition, overdispersion can 
148 arise for a variety of reasons, including aggregation (heterogeneity) in the data, or through

149 failing to measure important covariates, or include relevant interactions between covariates in

150 models (Hilbe 2011). However, multiple factors may interact with overdispersion to add further

151 bias to models of overdispersed data, including most notably the sample size of the datasets.

152 To date, relatively little is known in the ecological literature about the interaction between

153 overdispersion and sample size and how this affects parameter estimates. This is particularly

154 relevant to mixed effects models, where the number of grouping levels of a random intercept

155 term (e.g. number of populations) can greatly influence model accuracy. Low replication at the

156 level of the random effect grouping variable can mean there is not enough information to

157 estimate the variance among groups, especially if one employs 5 or fewer levels (Gelman and

158 Hill 2006 p247). Unfortunately, ecologists often fit factors containing fewer than 5 levels as

159 random effects (e.g. a random intercept for 'Year', Harrison et al 2013a,b), largely because

160 gathering 3 or 4 years of data in the laboratory or field represents an enormous amount of

161 work.

162 In order for OLRE to be considered a robust tool for modeling overdispersion in Binomial

163 data, they should yield accurate parameter estimates under a broad range of conditions,

164 including high overdispersion and low sample size. This paper investigates the influence of 3

165 specific variables on the accuracy of parameter estimates from mixed models: i) for a fixed

166 sample size, the influence of weak, moderate and strong overdispersion; ii) for strong

167 overdispersion, the influence of the level of replication of the random intercept term; and iii)

168 for strong overdispersion, the influence of Binomial sample size. For all three scenarios, I

169 consider overdispersion resulting from two mechanisms, using either a Beta-Binomial

170 distribution or an overdispersed Binomial distribution to generate the data (see equations

171 above). Finally, I use both OLRE and Beta-Binomial models to assess the relative performance of

172 each model type for a given scenario of overdispersion and sample size. Model performance is

173 assessed by i) quantifying the accuracy with which the models can recover estimates of $\beta_{\text {prey, }}$

$174 \mu_{\text {pop }}$ and $\sigma_{\text {pop }}^{2}$ (values fixed for all simulations); and ii) including a weak negative effect of $\beta_{\text {bodysize }}$

$175(-0.01)$, corresponding to roughly a $4 \%$ difference in reproductive success between the smallest

176 and largest individuals in the dataset, and quantifying the proportion of simulation replicates 
177 for a given scenario that incorrectly inferred a positive slope for $\beta_{\text {bodysize. Such an outcome is }}$ 178 important, because most ecological datasets likely contain variables of weak effect that are 179 'biologically relevant' to the organism(s) in question, but our ability to detect such effects in the 180 presence of overdispersion has received relatively little attention (but see Richards 2008).

181 
183

184

185

186

187

188

189

190

191

192

193

194

195

196

197

198

199

200

201

202

203

204

205

206

207

208

209

210

\section{METHODS}

\section{Data Generation}

I explored the consequences varying three key parameters in Binomial mixed models: i) the magnitude of overdispersion ('Overdispersion' Scenario), ii) the number of the levels of the random intercept term, ('Random Effect' Scenario) and iii) the Binomial sample size (number of trials per observation) ('Binomial Sample Size' Scenario). For each scenario, I simulated data from both an overdispersed Binomial distribution using eqn. 1-6 and a Beta-Binomial distribution using eqns 1-4 and 7-10 (see Introduction) to examine whether the accuracy of the mixed models also depended on the mechanism generating the overdispersion in the data. For the Overdispersion simulations, $\phi$ was set at $0.1,1$, or 2 for the Beta-Binomial data, and $\sigma_{\varepsilon}$ (specified as standard deviation, not variance $\sigma_{\varepsilon}^{2}$, in the R code) set to $0.1,1.5$ or 3 for the corresponding overdispersed Binomial data. A value of $\sigma_{\varepsilon}=\phi=0.1$ corresponds to weak overdispersion (model dispersion parameter $\sim 1.1$ ), whereas $\sigma_{\varepsilon}=3 / \phi=2$ corresponds to a model dispersion parameter of $\sim 2$. For all simulations parameter values were fixed at the following: $\mu_{\text {pop }}=-1 ; \sigma_{\text {pop }}=0.5 ; \beta_{\text {bodysize }}=-0.01 ; \beta_{\text {prey }}=0.6$. For the Overdispersion and Binomial Sample Size scenarios, I assumed 10 different populations had been sampled, each with a sample size of 20 individuals ( $n=200$ ). For the Random Effect scenario, the number of populations was set at 3,5 or 20 . Clutch size $\left(C_{i}\right)$ was fixed at 5 for the Overdispersion and Random Effect simulations, but was set to 2, 4 or 10 for the Binomial Sample Size scenario. Full details of the parameters used in each of the 3 scenarios are provided in Table 1.

\section{Model Fitting Simulations}

All simulations were coded in R v3.1.1 (R Core Team 2014). One thousand datasets were simulated for each set of three different parameter estimates for each of the three scenarios and data types (Beta-Binomial of overdispersed Binomial, see Table 1). For each dataset, I fitted model $\mathrm{m} 2$ containing an OLRE (see Introduction) in the Ime4 package and extracted parameter estimates for $\mu_{\mathrm{pop}}, \sigma_{\mathrm{pop}}, \beta_{\text {prey }}, \beta_{\text {bodysize }}$ and $\sigma_{\varepsilon}$ (the SD of the observation level random effect, 
211 'obs'). Following 1000 simulations, I calculated simulation means and 95\% quantiles for

212 parameters. I also calculated the proportion of models that falsely estimated the effect of body

213 size to be positive $\left(\beta_{\text {prey }}>0\right)$. Data for the proportion of models where $\beta_{\text {prey }}>0$ are presented as

214 means and bootstrapped 95\% confidence intervals for each parameter / data type combination.

215 I did not test for significant differences between mean values for each parameter.

216 For each of the three scenarios and two data types, I fitted a corresponding Bayesian

217 Beta-Binomial hierarchical model in JAGS (Plummer 2013) using the R package runjags

218 (Denwood 2014), following eqns 1-4 and 7-10 above. This resulted in four combinations of

219 data-generating process and statistical model used in analysis: Beta-Binomial data with OLRE,

220 Overdispersed Binomial data with OLRE, Beta-Binomial data with a Beta-Binomial Model, and

221 Overdispersed Binomial data with Beta-Binomial model. The Bayesian framework is extremely

222 flexible, meaning models following these equations can be easily specified, even though few

223 frequentist mixed model packages in R permit the fitting of Beta-Binomial models (but see 224 spaMM, Rousset and Ferdy 2014; glmmADMB, Fournier et al 2012). Models were run for 22520000 iterations with a thinning interval of 20 following a burnin of 2000 . Convergence was 226 assessed by running two parallel chains and calculating the Gelman-Rubin statistic, which was 227 below 1.05 for parameters, indicating convergence. Results are presented as posterior means 228 and $95 \%$ credible intervals for all parameters. I used uninformative Normal priors with mean 0 229 and precision 0.001 for $\mu_{\text {pop }}, \beta_{\text {prey }}$ and $\beta_{\text {bodysize; }}$ an uninformative uniform prior on the 230 interval $(0,10)$ for $\sigma$ pop, and an uninformative gamma prior with $a=b=0.001$ for $\phi$. To test 231 sensitivity of model output to choice of priors, I reran models where $\phi$ had a uniform prior on 232 the interval $(0,10)$ and $\sigma$ pop had a gamma prior where $a=b=0.001$. Results from both sets of 233 models were similar, suggesting limited sensitivity to prior specification.

234 Unlike frequentist models, their Bayesian equivalents are much more computationally 235 intensive and thus slower to run. Because of this, I generated only 1 dataset and ran 1 model 236 for each scenario / data type combination $(n=18)$. Both Bayesian and frequentist data are 237 plotted alongside one another in the figures, but it is important to note that the frequentist 238 data are the $95 \%$ intervals of the distribution of 1000 means for each parameter, whilst the 239 Bayesian data are the $95 \%$ credible intervals of 1000 samples from the parameter space for a 
240 single mean. Although they are slightly different quantities, the point of the comparison is to

241 assess the relative accuracy of a Beta-Binomial model compared to a Binomial model allowing

242 for overdispersion on the linear predictor i.e. containing observation-level random effects.

243 However because of the use of Bayesian analyses in these simulations, the type of model (OLRE

244 or Beta-Binomial) is therefore confounded with the fitting algorithm (Maximum Likelihood or

245 Bayesian, respectively). That is, frequentist methods may perform poorly in generalized mixed

246 models (Ferkingstad and Rue 2015), and Bayesian methods may perform slightly better, and

247 this may have little to do with the type of model. To test the sensitivity of the parameter

248 estimates to Beta-Binomial modeling philosophy, I reran the data simulations for highly

249 overdispersed Binomial $\left(\sigma_{\varepsilon}=3\right)$ and Beta-Binomial $(\phi=2)$ data with 10 populations, 20

250 individuals per population and a clutch size of 5 per individual. Instead of Bayesian Beta-

251 Binomial models, I fitted frequentist Beta-Binomial mixed models using the 'spaMM' package'

252 and extracted means and 95\% confidence intervals for parameters after 1000 simulations.

253 Model code for the Bayesian models, and all data simulations in the manuscript are

254 provided in Online Supplementary Information.

255 
257

258

259

260

261

262

263

264

265

266

267

268

269

270

271

272

273

274

275

276

277

278

279

280

281

282

283

\section{RESULTS}

Overdispersion

Weak overdispersion $\left(\sigma_{\varepsilon} / \phi=0.1\right.$ ) resulted in accurate parameter estimates for $\beta_{\text {prey, }} \mu_{\text {pop }}$ and $\sigma_{\text {pop }}$ for all four data/models combinations as expected (Figure 1). However, for both moderate and strong overdispersion, bias increased for all parameters when the data were generated from a Beta-Binomial distribution but analysed using OLRE (yellow circles, Fig. 1). Conversely, the Overdispersed Binomial / OLRE model did not suffer the same bias, although the standard error of all estimates increased in tandem with overdispersion (blue circles, Fig. 1). Betabinomial models performed well for both Beta-binomial and Overdispersed Binomial data (yellow and blue diamonds, Fig. 1), but were unable to accurately estimate $\sigma_{\text {pop }}$ when overdispersion was high ( $\sigma_{\varepsilon} / \phi=3$ / 2 respectively). Increasing overdispersion caused an increase in the proportion of models incorrectly inferring a positive slope for $\beta_{\text {bodysize }}$ for both types of data (Fig. 4A). Summary: OLRE are highly sensitive to the mechanism generating the overdispersion in the data, yielding large bias when applied to Beta-Binomial data. Parameter bias gets progressively worse as overdispersion increases.

\section{Number of Levels of The Random Effect}

For all four data/model combinations, the precision of the estimates increased as the number of levels of the random effect increased from 3 to 20 (Figure 2). This was expected, as a higher number of levels yields more information to estimate hierarchical modeling components such as $\sigma_{\text {pop. }}$. However, for the Beta-Binomial data / OLRE model, increasing the number of levels yielded both a consistently biased mean value for $\beta_{\text {prey, }}$ and increased precision around the biased mean (yellow circles, Fig. 2). OLRE models on Overdispersed binomial data (blue circles) performed generally well for both $\beta_{\text {prey }}$ and $\mu_{\text {pop. }}$. Conversely a Beta-Binomial model on Overdispersed Binomial data (blue diamonds, Fig. 2) tended to underestimate $\beta_{\text {prey. }}$ For all four data/model combinations, estimates of $\sigma_{\text {pop }}$ were highly imprecise when only 3 populations 
284 were considered. This was especially true for both Beta-Binomial models (yellow and blue

285

286

287

288

289

290

291

292

293

294

295

296

297

298

299

300

301

302

303

304

305

306

307

308

309

310

311

312

diamonds, Fig. 2). There was still a large degree of bias when $n=5$, especially when OLRE models were applied to Beta-Binomial data (yellow circles and diamonds). The proportion of models recovering $\beta_{\text {bodysize }}>0$ tended to decrease as the $n$ increased, but only by $5 \%$ on average, and there appeared to be no differences in proportion depending on the kind of overdispersion generated in the data. Increasing the sample size to 200 but using only 3 populations (67 individuals per population) still resulted in biased parameter estimates, especially for Beta-Binomial models (Table 2a), suggesting it is random effects sample size and not total sample size driving this pattern. Summary: Higher replication of the random effects results in more precise estimates, but cannot fix the bias caused by overdispersion when using OLRE for some data types. All models perform poorly when the number of populations is 3 , especially for $\sigma_{\text {pop, }}$ suggesting there is no modeling 'fix' for poor replication of the random effect.

\section{Binomial Sample Size}

Of the three scenarios considered, Binomial sample size had the smallest effect on parameter accuracy and precision. As with the Random Effects scenario, increasing Binomial sample size did not remedy the bias in $\beta_{\text {prey }}$ caused by overdispersion, but did yield slightly higher precision (yellow circles, Fig. 3). Estimates for all other parameters were similar irrespective of Binomial sample size and type of overdispersion (blue circles, yellow diamonds and blue diamonds, Fig. 3 ), and the proportion of models where $\beta_{\text {bodysize }}>0$ were fairly constant across all values tested (Fig. 4C). Summary: OLRE models on Beta-Binomial data continue to perform poorly in the presence of overdispersion, irrespective of the Binomial sample size. All other models performed equally well, and there was evidence suggesting that even over the narrow range of sample sizes tested (2-10), precision increased with sample size.

\section{A Comparison of Frequentist and Bayesian Beta-Binomial Estimates}

Model estimates from the Beta-Binomial models were very similar irrespective of whether they were from frequentist or Bayesian models (Fig. 1 and Table 2b). Interestingly, both the frequentist and Bayesian models suggested that using Beta-Binomial models on Overdispersed 
313 Binomial data leads to underestimating the values of both of $\mu_{\text {pop }}$ (middle pane, Fig. 1; Table 2c)

314 and $\beta_{\text {prey }}$ (left pane, Fig. 1; Table 2 b). Collectively these results suggest the discrepancies in

315 parameter estimates observed between OLRE and Beta-Binomial models were not simply due

316 to using either Bayesian or frequentist methods, but reflected a genuine difference in ability of

317 certain models to handle certain types of overdispersion.

318 


\section{DISCUSSION}

321 Using a simulation approach, I have investigated both the ability of observation-level random

322 effects to recover accurate parameter estimates under various degrees of overdispersion in

323 Binomial models, and whether the performance of OLRE in mixed models is consistent across

324 multiple types of overdispersion. In addition, I have examined how model performance changes

325 in the presence of overdispersion when both the sample size of random effects (number of

326 levels) and Binomial sample size is low for both OLRE and Beta-Binomial models. In general,

327 OLRE models performed poorly when fitted to Beta-Binomial data, and this effect was

328 particularly pronounced when the number levels of the random effect was $\leq 5$, or the Binomial

329 sample size was small. In all cases, increasing the number of levels of the random effect or

330 Binomial sample size failed to remedy the bias in the estimates caused by overdispersion. Here I

331 discuss the implications of these results for choosing OLRE as a suitable tool to model

332 overdispersion in ecological data.

333

334 The ability of OLRE to cope with overdispersion depends on the process generating the 335 overdispersion

336 This study has shown that the ability of OLRE to recover accurate parameter estimates in overdispersed mixed models depends on the process generating the overdispersion in the dataset. For overdispersion generated by adding random noise to the linear predictor (overdispersed Binomial data), the model recovered accurate mean estimates for slopes, intercepts and variance components at all levels of overdispersion, although the precision of the estimates declined (increasing standard errors) as overdispersion increased. Conversely, for data generated using a Beta-Binomial process, parameter estimates became increasingly more biased as overdispersion increased, leading to inflated estimates of effect size for the variables

344 of interest. The implications of these results are that OLRE may not be a robust tool for dealing with overdispersion in Binomial mixed models because the researcher is unlikely to know which process generated the overdispersion in the first instance, meaning it is unclear if the parameter estimates are trustworthy. More worryingly, that the use of OLRE can lead to 
348 inflated effect sizes may result in researchers concluding that variables under investigation are 349 highly influential, when in fact their effect sizes may be more modest. These patterns were not 350 observed in the Beta-Binomial models, irrespective of data type, although the confidence 351 intervals did also increase in concert with overdispersion. The relative utility of Beta-Binomial 352 models over OLRE models is discussed below.

353

354 Increasing random effect sample size increases precision, even for biased estimates

355 For all model/data combinations, increasing the number of populations measured from 3 to 20

356 greatly increased the precision of estimates for all parameters, reflected by smaller $95 \%$

357 confidence intervals. Importantly, this result held even when controlling for total sample size,

358 demonstrating that higher replication within populations cannot compensate for fitting a 359 population random intercept term with only 3 levels. Arguably, parameter precision was also 360 poor when using 5 populations, especially for the variance component $\sigma_{\text {pop }}$. This corroborates

361 the general rule of thumb that random intercept terms should ideally contain more than 5

362 levels in order to yield accurate estimates and good model performance (Gelman and Hill

363 2006), especially when overdispersion is present. The key result of the random effects

364 simulations is that when using OLRE on Beta-Binomial data, increasing sample size of the 365 random effect does nothing to remedy the bias in slope estimates for effects such as $\beta_{\text {prey }}$.

366 Instead, increasing the number of levels of the random effect simply makes one more certain of

367 the accuracy of the estimates by decreasing the $95 \%$ confidence intervals, even when the mean

368 estimate is biased. This is worrying, as well-replicated studies studying 10 s to 100 s of 'groups'

369 (be they populations, genetic lines or sampling locations etc.) may recover highly precise

370 estimates for parameters that are highly inflated with respect to their true value. In addition, it

371 means one cannot use enormous standard errors as diagnostic evidence for suspicious behavior

372 of OLRE (i.e. for $\beta_{\text {prey }}$ when $n$ populations $=3$ ) because these are likely to change with sample 373 size.

374

375 Parameter estimates are largely similar irrespective of Binomial Sample Size 
376 Binomial sample size had the smallest influence on model behavior of the three scenarios

377 tested. There was some indication that parameter accuracy increased with Binomial sample

378 size, but these effects were modest for most data/model combinations, especially when

379 compared to the relatively large influence of random effect sample size (see above). Such a

380 result is intuitive, as one would expect total sample size to be more influential than simply the

381 sample size of a single observational unit. That model precision is similar irrespective of

382 maximum sample size is encouraging, particularly as researchers in the fields of ecology and

383 evolution deal with an enormous range in sample sizes e.g. analyzing the hatching success of a

384 bird that produces only 2 eggs at a time (Harrison et al 2013b) or an insect that produces 100s

385 of eggs at a time (Tyler et al 2014). Collectively these results suggest that model precision need

386 not be sacrificed if working on organisms with life history characteristics such as small clutch

387 size or low fecundity.

388

389 Are Beta-Binomial models more robust than OLRE models for Binomial data?

390 A persistent pattern in the results shown here is that OLRE perform poorly for Beta-Binomial

391 data, yet Beta-Binomial models tend to perform well across both Beta-Binomial and

392 Overdispersed Binomial data. This does not mean that OLRE models are unsuitable for

393 modeling overdispersion, simply that one must interpret initial model results with caution and

394 examine the suitability of OLRE for a particular dataset. The most straightforward way to probe

395 the robustness of OLRE model results would be to compare parameter estimates from a given

396 OLRE model with the Beta-Binomial equivalent. There were dramatic differences in estimates

397 for the $\beta_{\text {prey }}$ slope between models types for the Beta-Binomial data, yet very little difference

398 for the corresponding overdispersed Binomial data, and so the comparative approach should

399 readily identify potential problems with OLRE.

400 When performing model comparison, whether one uses a frequentist statistical

401 package, or codes the model manually in a Bayesian framework in JAGS appears to be a matter

402 of preference, as these two approaches yielded similar results in the current study. Although

403 Bayesian methods do have several advantages over frequentist methods (e.g. Ellison 2004; Kery

404 2010), in many cases they recover similar parameter estimates to frequentist models when 
405 uninformative priors are used (e.g. Kery 2010). In support of this, sensitivity analyses presented 406 in this paper suggest that model results are similar whether one uses a Bayesian or a

407 frequentist Beta-Binomial model, meaning choice of model rather than of statistical philosophy 408 is the more important driver here. However, I would caution that I only repeated the 409 frequentist Beta-Binomial simulations for a limited subset of cases (high overdispersion, 10 410 populations, 20 individuals per population), and so it should not be assumed that frequentist 411 and Bayesian approaches would agree in every case (see also examples in Kery 2010). A final 412 caveat for these results is that the data generated in this study are 'ideal' in so far as they are 413 perfectly balanced across populations (identical numbers of individuals per population). In 414 reality this is unlikely to hold, as ecological datasets often contain poorly represented groups 415 with far lower sample sizes than others e.g. years with poor breeding success and limited data 416 on clutch size (e.g. Harrison et al 2011; 2013b). How imbalance in sample size affects model 417 estimates in the presence of overdispersion for two models with identical number of levels of 418 the random intercept term warrants further investigation.

419 Superficially, it appears that Beta-Binomial models perform better than OLRE models in 420 most cases, and so a natural inclination would be to simply use Beta-Binomial models for any 421 kind of overdispersed Binomial data. However, results from this study indicated that Beta422 Binomial models can underestimate slope values (e.g. the value of $\beta_{\text {prey }}$ ), whereas the 423 corresponding OLRE model does not. Thus, Beta-Binomial models do not universally 424 outperform OLRE models, and one should not sacrifice OLRE from the set of tools available to 425 deal with overdispersed Binomial data. Both Beta-Binomial and OLRE models estimate an 426 overdispersion parameter that can reveal a biological cause underlying aggregation/non427 independence of probabilities in the dataset (e.g. Beta-Binomial models, Hilgenboeker et al 428 2008; OLRE models, Elston et al 2001), and the size of the aggregation parameter is informative 429 and comparable across studies (Richards 2008). Beta-binomial models are frequently employed 430 in the ecological literature to model non-independence among probabilities (e.g. Hughes and 431 Madden 1993; Lee and Nelder 1996; Clark 2003; Richards 2008) and may be less prone to 432 overfitting than the corresponding OLRE models, which may explain why OLRE models 433 performed poorly for Beta-Binomial data. Indeed, the dispersion parameter of models 
434 containing OLRE frequently collapses to $<0.5$ (data not shown), suggesting the addition of OLRE 435 replaces overdispersion with underdispersion, which can be equally as problematic (Zuur et al 436 2009).

437

438

439 Summary

440 Observation-level random effects provide a simple means to control overdispersion that 441 can be easily implemented in mixed effects model packages. However, it is clear that their use 442 may not be appropriate in all cases. Results from models containing OLRE should be carefully 443 inspected, and where possible corroborative evidence should be sought from alternative 444 modeling approaches such as (Bayesian) Hierarchical Beta-Binomial models to quantify 445 agreement between parameter estimates and ensure the conclusions drawn from such 446 analyses are robust. Finally, one should avoid fitting random intercept terms to models when 447 the random term contain $<5$ levels, especially in the presence of overdispersion, as parameter 448 estimates become unreliable irrespective of modeling approach. One should also interpret 449 model results with caution when the random effect sample size is large (e.g. $>20$ ), because 450 models with OLRE can yield inaccurate but precise (small confidence intervals) slope estimates 451 under certain scenarios that may give the false impression of that model having performed 452 well.

453

454 REFERENCES

455

456 457

Bates D, Maechler M, Bolker B, Walker S. 2014. Ime4: Linear mixed-effects models using Eigen and S4. R package version 1.1-6. http://CRAN.R-project.org/package=Ime4

458

459 460

Bielby J, Donnelly CA, Pope LC, Burke T, Woodroffe R. 2014. Badger responses to small-scale culling may compromise targeted control of bovine tuberculosis. Proceedings of the National Academy of Sciences,

461 111: 9193-9198.

462 
463

464

465

466

467

468

469

470

471

472

473

474

475

476

477

478

479

480

481

482

483

484

485

486

487

488

489

490

491

492

493

494

495

496

497

Bolker BM, Brooks ME, Clark CJ, Geange SW, Poulsen JR, Stevens MHH, White JSS. 2009. Generalized linear mixed models: a practical guide for ecology and evolution. Trends in ecology and evolution, 24:127-135.

Denwood MJ. 2014. runjags: An R package providing interface utilities, parallel computing methods and additional distributions for MCMC models in JAGS. Journal of Statistical Software. http://cran.rproject.org/web/packages/runjags/

Ellison AM. 2004. Bayesian inference in ecology. Ecology Letters, 7: 509-520.

Elston DA, Moss R, Bouliner T, Arrowsmith C, Lambin X. 2001. Analysis of aggregation, a worked example: number of ticks on red grouse. Parasitology, 122:563-569.

Ferkingstad, E., Rue, H. (2015). Improving the INLA approach for approximate Bayesian inference for latent Gaussian models. arXiv preprint arXiv:1503.07307.

Forstmeier W, Schielzeth H. 2011. Cryptic multiple hypotheses testing in linear models: overestimated effect sizes and the winner's curse. Behavioral Ecology and Sociobiology 65:47-55.

Fournier DA, Skaug HJ, Ancheta J, lanelli J, Magnusson A, Maunder M, Nielsen A, Sibert J. 2012. “AD Model Builder: using automatic differentiation for statistical inference of highly parameterized complex nonlinear models. Optim. Methods Softw.,,27:233-249.

Gelman A, Hill J. 2006. Data analysis using regression and multilevel/hierarchical models. Cambridge University Press, Cambridge, UK.

Harrison XA, York JE, Cram DL, Hares MC, Young AJ. 2013a. Complete reproductive skew within white-browed sparrow weaver groups despite outbreeding opportunities for subordinates of both sexes. Behavioral Ecology and Sociobiology, 67:1915-1929.

Harrison XA. 2014. Using observation-level random effects to model overdispersion in count data in ecology and evolution. PeerJ 2:e616.

Harrison XA, Hodgson DJ, Inger R, Colhoun K, Gudmundsson GA, McElwaine G, Bearhop S. 2013b. Environmental conditions during breeding modify the strength of mass-dependent carry-over effects in a migratory bird. PLOS One 8: e77783. 
Harrison XA, Bearhop S, Inger R, Colhoun K, Gudmundsson GA, Hodgson D, Tregenza T. 2011. Heterozygosityfitness correlations in a migratory bird: an analysis of inbreeding and single-locus effects. Molecular

501 Ecology, 20:4786-4795.

502

503

Hilbe JM. 2011. Negative Binomial Regression. 2nd Edition. Cambridge University Press, Cambridge, UK

504

505

Hilgenboecker K, Hammerstein P, Schlattmann P, Telschow A, Werren JH 2008. How many species are infected

506 with Wolbachia?-a statistical analysis of current data. FEMS Microbiology Letters, 281: 215-220.

507

508

509

Hughes G, Madden LV 1993. Using the beta-binomial distribution to describe aggegated patterns of disease

510

511

Kery M. 2010. Introduction to WinBUGS for Ecologists. Academic Press, Burlington, MA.

512

513 Lee Y, Nelder JA (1996). Hierarchical generalized linear models. Journal of the Royal Statistical Society. Series B $514 \quad$ (Methodological), 619-678.

515

516

Link WA, Barker RJ. 2006. Model weights and the foundations of multimodel inference. Ecology 87:2626-2635.

517

518

Martin TG, Wintle BA, Rhodes JR, Kuhnert PM, Field SA, Low-Choy SJ, Tyre AJ, Possingham HP. 2005. Zero tolerance

519 ecology: improving ecological inference by modelling the source of zero observations. Ecology Letters

520 8:1235-1246.

521

522 McCarthy MA. 2007. Bayesian Methods for Ecology. Cambridge University Press. Cambridge, UK.

523

524

Millar RB. 2009. Comparison of Hierarchical Bayesian Models for Overdispersed Count Data using DIC and Bayes'

525 Factors. Biometrics 65:962-969.

526

527 Plummer M. 2013. JAGS: Just Another Gibbs Sampler, Version 3.4. 0. URL http://mcmc-jags. sourceforge. net. 528

529 R Core Team. 2014. R: A language and environment for statistical computing. R Foundation for Statistical 530 Computing, Vienna, Austria. URL http://www.R-project.org/.

531 
532 Rousset F, Ferdy J. 2014. Testing environmental and genetic effects in the presence of spatial autocorrelation.

533 Ecography 37: 781-790.

534

535 Spiegelhalter DJ, Best NG, Carlin BP and Van Der Linde A. 2002. Bayesian measures of model complexity and fit.

536 Journal of the Royal Statistical Society: Series B (Statistical Methodology), 64:583-639.

537

538 Tyler F, Harrison XA, Bretman A, Veen T, Rodríguez-Muñoz R, Tregenza T. 2013. Multiple post-mating barriers to 539 hybridization in field crickets. Molecular Ecology, 22:1640-1649.

540

541 Zuur AF, leno EN, Walker NJ, Saveliev AA, Smith GM. 2009. Mixed Effects Models and Extensions in Ecology with R. $542 \quad$ Springer, New York.

543

544 Williams DA. 1982. Extra-binomial variation in logistic linear models. Applied statistics, 31:144-148.

545

546 


\section{Figure $\mathbf{1}$ (on next page)}

Effect of Varying Degrees of Overdispersion on Parameter Estimation

Parameter estimates and 95\% intervals for 3 levels of overdispersion under 4 combinations of overdispersion and model type. Yellow circles: Beta-Binomial overdispersion and an observation-level random effect (OLRE) model; blue circles: Overdispersed Binomial data and OLRE model; yellow diamonds: Beta-Binomial data and a Beta-Binomial model; blue diamonds: Overdispersed Binomial data and a Beta-Binomial model. Beta-Binomial data were analysed using Bayesian Hierarchical Beta-Binomial mixed models and so error bars are $95 \%$

credible intervals. ' $\beta_{\text {prey }}$ ' slope parameter for effect of number of prey items consumed; ' $\mu_{\text {pop }}$ ' mean value of population random intercept term; ' $\sigma_{\mathrm{pop}}$ ' standard deviation of population random effect. $X$ axis labels refer to the overdispersion parameters for each model type: Overdispersed Binomial models, $\sigma_{\varepsilon}$, the standard deviation of a random effect with mean 0 added to the linear predictor; Beta-Binomial models, $\varphi$, the dispersion parameter for the Beta-Binomial mixture distribution. 

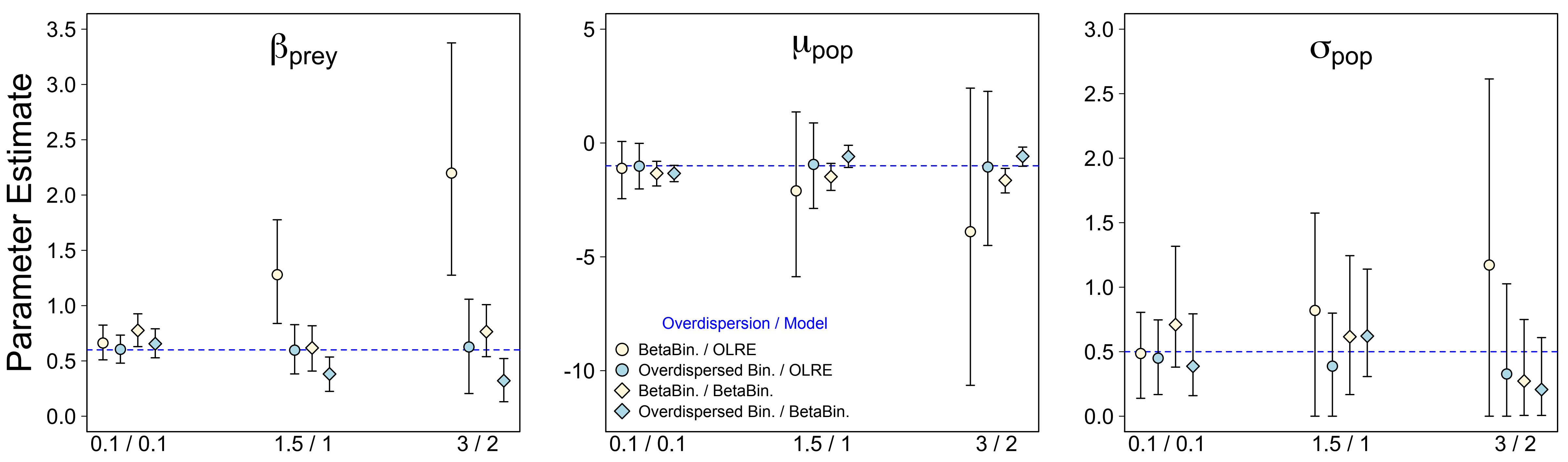

$\sigma_{\varepsilon} / \phi$ 


\section{Figure 2 (on next page)}

Effect of varying sample size of the random intercept term (number of populations) on parameter estimates in the presence of overdispersion

Parameter estimates and $95 \%$ intervals for 3 different levels of replication of the random intercept term under 4 combinations of overdispersion and model type. Yellow circles: BetaBinomial overdispersion and an observation-level random effect (OLRE) model; blue circles: Overdispersed Binomial data and OLRE model; yellow diamonds: Beta-Binomial data and a Beta-Binomial model; blue diamonds: Overdispersed Binomial data and a Beta-Binomial model. Beta-Binomial data were analysed using Bayesian Hierarchical Beta-Binomial mixed models and so error bars are $95 \%$ credible intervals. ' $\beta_{\text {prey }}$ ' slope parameter for effect of number of prey items consumed; ' $\mu_{\mathrm{pop}}$ ' mean value of population random intercept term; ' $\sigma_{\mathrm{pop}}$ ' standard deviation of population random effect. For all simulations $\sigma_{\varepsilon}$ was set to 3 for Overdispersed Binomial models, and $\varphi$ set to 2 for Beta-Binomial models. 

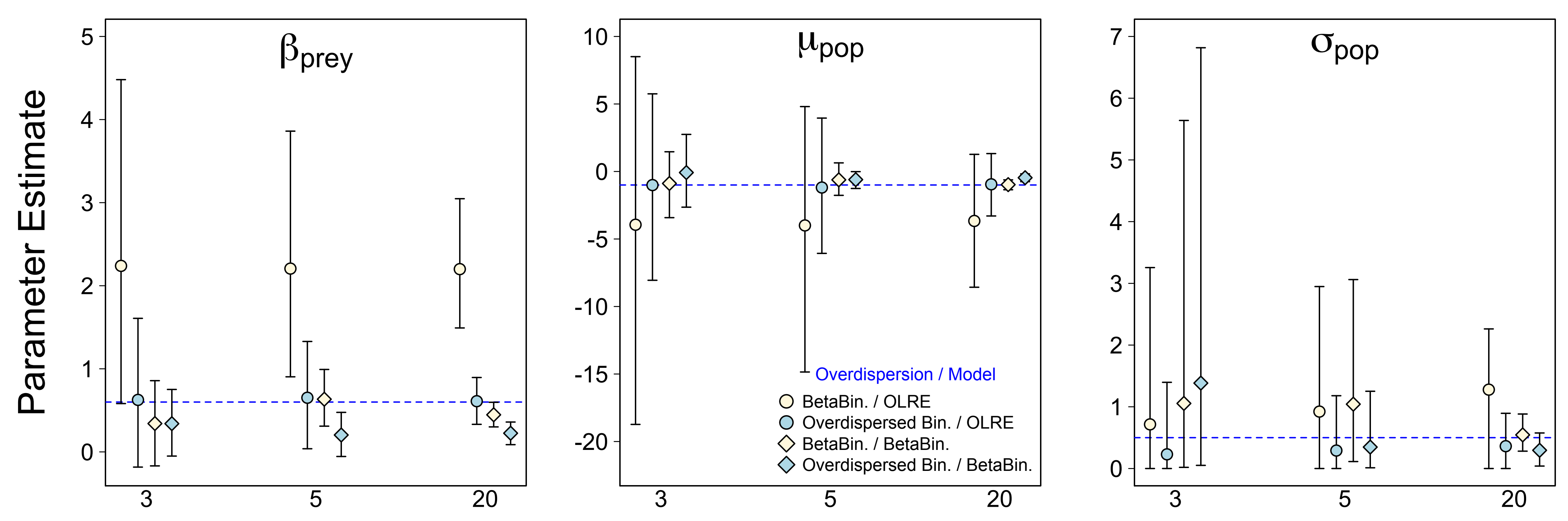

n Populations for Random Intercept 


\section{Figure 3 (on next page)}

Effect of Binomial sample size on accuracy of parameter estimates in the presence of overdispersion

Parameter estimates and 95\% intervals for 3 different Binomial sample sizes (clutch size) under 4 combinations of overdispersion and model type. Yellow circles: Beta-Binomial overdispersion and an observation-level random effect (OLRE) model; blue circles:

Overdispersed Binomial data and OLRE model; yellow diamonds: Beta-Binomial data and a Beta-Binomial model; blue diamonds: Overdispersed Binomial data and a Beta-Binomial model. Beta-Binomial data were analysed using Bayesian Hierarchical Beta-Binomial mixed models and so error bars are $95 \%$ credible intervals. ' $\beta_{\text {prey }}$ ' slope parameter for effect of number of prey items consumed; ' $\mu_{\mathrm{pop}}$ ' mean value of population random intercept term; ' $\sigma_{\mathrm{pop}}$ ' standard deviation of population random effect. For all simulations $\sigma_{\varepsilon}$ was set to 3 for Overdispersed Binomial models, and $\varphi$ set to 2 for Beta-Binomial models. 

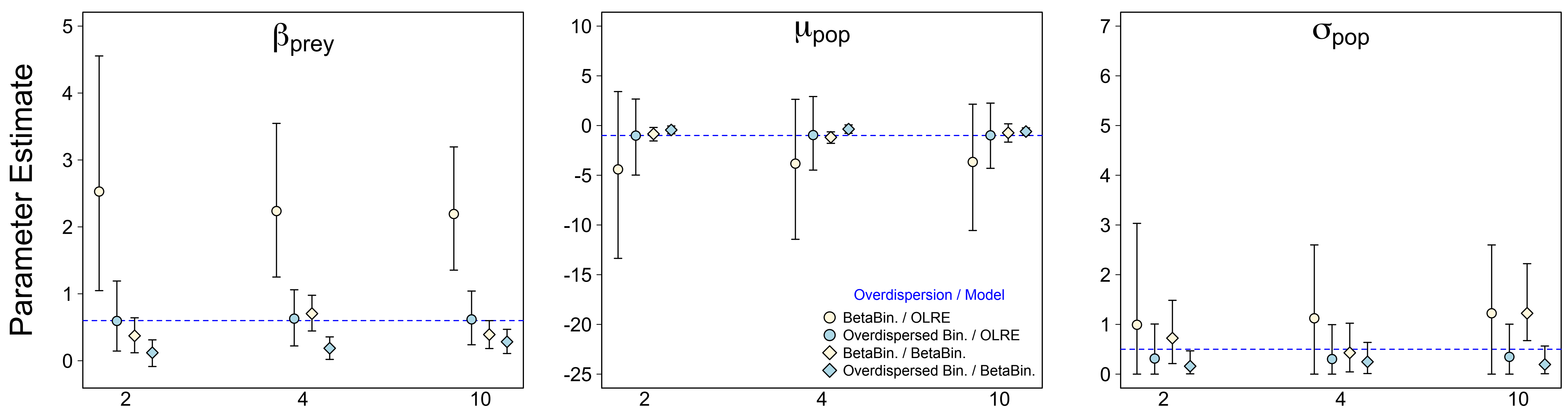

Binomial Sample (Clutch) Size 
Figure 4 (on next page)

Proportion of models after 1000 simulations incorrectly estimating the weakly negative slope of the body size parameter to be positive

Bars are mean and $95 \%$ confidence intervals following 1000 simulations of either BetaBinomial (yellow bars) or Overdispersed Binomial (blue bars) data and analysed with OLRE models. a) the influence of increasing levels of overdispersion; b) the influence of increasing the replication of the random intercept term for population; c) the influence of increasing the Binomial sample size (total clutch). 


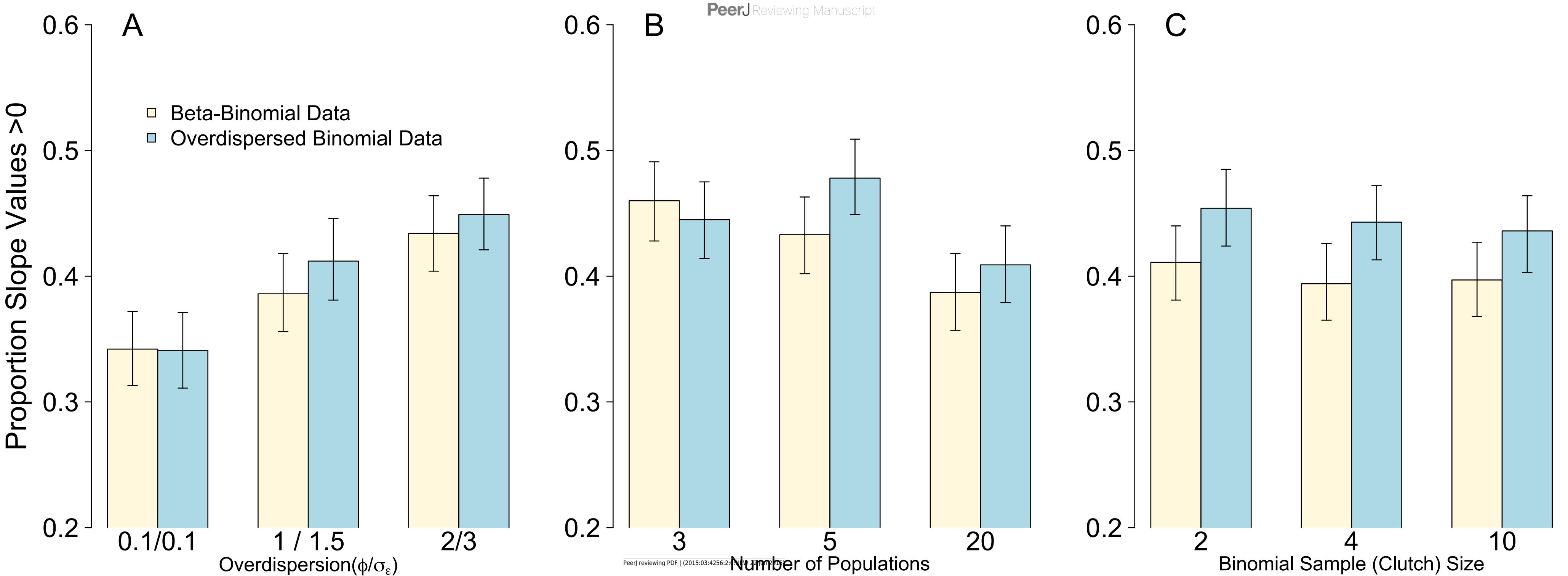




\section{Table $\mathbf{1}_{\text {(on next page) }}$}

Parameter values for simulation scenarios employed in the study

' $\varphi$ ' overdispersion parameter for Beta-Binomial models; ' $\sigma_{\varepsilon}$ ' overdispersion parameter for Overdispersed Binomial models; ' $n$ trials' Binomial sample size (maximum clutch) for simulations, equivalent to $C_{i}$ in equation 1 ; ' $n$ individuals': number of individuals per simulated population; ' $n$ populations': number of populations simulated for each dataset, and fitted as a random intercept term in all models, referred to as random effect sample size. Values for the variable under investigation in each scenario are shown in bold. 


\begin{tabular}{|c|c|c|c|c|}
\hline & $\begin{array}{c}\text { Overdispersion } \\
\phi / \sigma_{\varepsilon}\end{array}$ & $\begin{array}{c}\mathrm{n} \text { trials } \\
\text { (clutch size) }\end{array}$ & $\begin{array}{c}n \\
\text { individuals }\end{array}$ & $\begin{array}{c}n \\
\text { populations }\end{array}$ \\
\hline \multicolumn{5}{|c|}{ Overdispersion } \\
\hline 1 & $0.1 / 0.1$ & 5 & 20 & 10 \\
\hline 2 & $1 / 1.5$ & 5 & 20 & 10 \\
\hline 3 & $2 / 3$ & 5 & 20 & 10 \\
\hline \multicolumn{5}{|c|}{ Levels of Random Effect } \\
\hline 1 & $2 / 3$ & 5 & 20 & 3 \\
\hline 2 & $2 / 3$ & 5 & 20 & 5 \\
\hline 3 & $2 / 3$ & 5 & 20 & 20 \\
\hline \multicolumn{5}{|c|}{ Binomial Sample Size } \\
\hline 1 & $2 / 3$ & 2 & 20 & 10 \\
\hline 2 & $2 / 3$ & 4 & 20 & 10 \\
\hline 3 & $2 / 3$ & 10 & 20 & 10 \\
\hline
\end{tabular}

1 


\section{Table 2 (on next page)}

Model results investigating the effects of total sample size and model type on parameter estimates

a) Parameter values and $95 \%$ confidence intervals for 1000 simulations of data where 3 populations were simulated each with a sample size of $67(n=201)$ and analysed with OLRE models. Results are highly similar to when 3 populations are simulated each with a sample size of 20 ( $n=60$, see Figure 2), suggesting it is replication of the random effects and not total sample size driving the poor performance of models. ' $\beta_{\text {prey }}$ ' slope parameter for effect of number of prey items consumed; ' $\beta_{\text {bodysize }}$ ' slope parameter for effect of individual body size; ' $\mu_{\text {pop }}$ ' mean value of population random intercept term; ' $\sigma_{\text {pop }}$ ' standard deviation of population random effect. True simulated values are shown in parentheses. 'Beta-Binomial Data' refer to data generated from a Beta-Binomial mixture model with dispersion parameter $\varphi=2$; 'Overdispersed Binomial Data' refers to data generated by adding random noise to the linear predictor of a Binomial model on the link scale, from a Normal distribution with mean 0 and standard deviation $\sigma_{\varepsilon}=3$. b) Parameter values and $95 \%$ confidence intervals for 1000 simulations of overdispersed data analysed with frequentist Beta-Binomial models. Results are highly similar to their Bayesian equivalents (see Figure 1), suggesting it is the mechanism generating the overdispersion in the data that results in poor parameter estimates and not modelling philosophy (frequentist/maximum likelihood vs Bayesian). 


\begin{tabular}{ccccc} 
& & \multicolumn{2}{c}{$\begin{array}{c}c \\
\text { a) }\end{array}$} & \multicolumn{2}{c}{ Data } & \multicolumn{2}{c}{ Overdispersed Binomial } \\
Data
\end{tabular}

